\title{
NUEVO GENERO Y DOS ESPECIES NUEVAS DE ANTHICIDAE PARA CHILE (COLEOPTERA)
}

\author{
NEW GENUS AND TWO NEW SPECIES OF ANTHICIDAE \\ FOR CHILE (COLEOPTERA)
}

\author{
Tomás Moore ${ }^{1} \&$ Pedro Vidal ${ }^{2}$ \\ 1El Vergel 2245, Depto. 28-D, Santiago, Chile. Email: tmoore@vtr.net \\ ${ }^{2}$ Pontificia Universidad Católica de Chile. Facultad de Medicina, Lira 44, Santiago, Chile. \\ Email: pvidalg@puc.cl
}

\begin{abstract}
RESUMEN
Se describe un nuevo género, Protoanthicus, con dos especies nuevas: P. marziae n. sp. y P. valenciai n. sp. para Chile (Anthicidae, Coleoptera).
\end{abstract}

Palabras Claves: Anthicidae, Chile, taxonomía.

\section{ABSTRACT}

A new genus Protoanthicus and two new species, P. marziae sp. n. and P. valenciai sp. n. are described for Chile (Anthicidae, Coleoptera).

KEYwords: Chilean anthicids, taxonomy.

\section{INTRODUCCION}

La familia Anthicidae está presente en Chile desde la III Región hasta la IX Región. En la Patagonia argentina existe la especie Anthicus torquatus Werner, que ha sido colectada en Santiago (espe-címenes sin duda introducidos). Los 6 géneros conocidos que están presentes en Chile son: Anthicus Paykull, Chileanthicus Werner, Ischyropalpus Bonadona, Vacusus Werner, Mitraelabrus Solier y Copobaenus Fairmaire \& Germain.

Germain en 1911 propuso el género Licancurus (nomen nudum) para incorporar el material de Coquimbo (según ejemplar etiquetado por Germain y depositado en el MNNC), pero nunca describió el género ni tampoco la especie, pre- sentando diferencias con los géneros existentes en Chile y algunas características primitivas que permiten fácilmente su diferenciación (Abdullah 1969). Como resultado del estudio de material recolectado en el Cerro Santa Inés, IV Región, se separaron los ejemplares de Antícidos que no pertenecían a ningún género de los citados arriba.

\section{MATERIALES Y METODO}

Se estudió material de Anthicidae existente en la colección del Dr. Pedro Vidal, proveniente del Cerro Santa Inés, IV Región y del Parque Nac. Fray Jorge, IV Región y el existente en el Museo Nacional de Historia Natural del P.N. Fray Jorge y se comparó material de la familia Anthicidae de las colecciones indicadas a continuación: 
MNNC: Museo Nacional de Historia Natural, Santiago, Chile.

UMCE: Instituto de Entomología, Universidad Metropolitana de Ciencias de la Educación.

SAGV: Servicio Agrícola Ganadero, Valparaíso, Chile.

PVGH: Colección Sr. Pedro Vidal G-H., Santiago, Chile.

VMDC: Colección Sr. Víctor Manuel Diéguez, Santiago, Chile.

FRC: Colección Sr. Francisco Ramírez, Santiago, Chile.

JVJC: Colección Sr. Jorge Valencia, Valparaíso, Chile.

\section{RESULTADOS}

Del estudio del material de las colecciones antes citadas, se pudo determinar que el material de la V Región se trataba de una especie con caracteres similares al material de la IV Región ya citado . Las genitalias de los machos de las especies nuevas propuestas difieren claramente entre si y del resto de las especies dadas para Chile (Werner 1974 y Elgueta \& Arriagada 1989), y dado que poseen un fuerte surco pronotal mediano, las asemejan a las especies polinésicas del género Stricticomus (Uhmann 1995) y neárticas del género Formicomus Ferté-Sénectère, (Werner 1958 \& Ross \& Arnett 1973).

De los materiales estudiados se propone un género nuevo: Protoanthicus n. gen., y dos especies nuevas: P. marziae $\quad$ sp. n. y P. valenciaee sp. $\mathrm{n}$.

Las características primitivas que menciona Abdullah 1969 y que dan origen al nombre del nuevo género, se refieren a: ojos no marginados y con gruesa celación; Antenas con 11 segmentos, siendo los 3 apicales no notoriamente alargados; cuello ancho; pronoto sin flange anterior; abdomen del macho sin apéndices.

\section{Género Protoanthicus n. gen.}

Diagnosis: Especie tipo: P. marziae n. sp.

CABEzA: Marrón-rojizo a marrón claro, según los individuos, ancha, dos veces el ancho del cuello y casi tan ancha como la parte anterior del pronoto; puntuación gruesa homogénea, sedas leonadas reclinadas, ojos pequeños subcirculares, gruesamente facetados, no marginados. Antenas gruesas, pilosas, palpo maxilar con segmento apical securiforme (laminado) bien desarrollado.Cuello: fuerte, ancho, ocupando el tercio central del ancho pronotal anterior.

PRONOTO: Mismo color que la cabeza, más largo que ancho, gruesa y densamente puntuado, surco mediano longitudinal entero o en los dos tercios basales, sin flange anterior, pilosidad leonada reclinada no densa.

ELITRos: Misma coloración que el pronoto, con zona apical más clara, desteñida, pilosidad leonada reclinada, puntuación gruesa y homogénea, costillas inexistentes, más cortos que el abdomen, ápice truncado y sin denticulación. Alas vestigiales, no voladoras.

FAZ INFERIOR: Marrón-rojizo a marrón claro brillante, pilosa; cavidades de las coxas anteriores internamente abiertas y cerradas externamente, abdomen con 5 segmentos visibles marrón claro, fina y densamente puntuados; coxas posteriores separadas, patas marrón-rojizo a testáceas con garras simples.

ETimología: Nombre derivado de sus destacadas características primitivas señaladas en su descripción: Proto = Inicial, primigéneo y anthicus de la familia Anthicidae.

\section{Protoanthicus marziae n. sp.}

(Figs, 1-2)

Diagnosis (Holotipo o ):

Largo 3,2 mm, ancho 1,2 mm. Especie muy notable por su colorido marrón brillante y surco pronotal mediano (Fig. 1).

CABEZA: Marrón-rojizo, menos oscuro que la zona discal elitral, subplana, puntuación densa homogénea, sedas leonadas reclinadas separadas, antenas gruesas que sobrepasan la base pronotal con los 4 artículos apicales, segundo antenito más corto que el tercero; ojos redondos pequeños fuertemente facetados, frente estrechada como hocico.

Pronoto. Mismo color que la cabeza, menos brillante que los élitros, algo más oscuro en los márgenes laterales, más largo que ancho, poco dilatado lateral- 
mente en la zona basal, lados casi subrecto, surco mediano longitudinal poco profundo, en los dos tercios basales, más notorio en las $q$ ㅇ, sin formación de lóbulos resaltados, puntuación gruesa y densa, pilosidad leonada rala, más erecta hacia los lados.

ELITROs: Marrón-rojizo oscuro, brillante con sutura y zona apical más clara, puntuación irregularmente dispuesta, moderadamente densa, costillas inexistentes, lados paralelos en su mayor parte, callo humeral no desarrollado, ápice truncado subredondeado oblicuamente, pubescencia densa leonada, reclinada. Alas vestigiales.

FAZ INFERIOR: Marrón-rojizo oscuro, brillante, puntuación densa, más fina que la del pronoto, pilosidad leonada más densa que la del pronoto, reclinada; segmentos abdominales marrón claro brillante, pubescentes, similar a la del esterno, borde apical de los segmentos más oscuro, epipleuras elitrales finamente ribeteadas marrón oscuro, irregularmente puntuadas, disminuyendo su ancho gradualmente hacia el ápice, patas marrón-rojizo, algo más claras.

EDEAGo: Largo, delgado, parámeros soldados, estrechado hacia el ápice desde el cuarto anterior y suavemente acinturado en los 3/4 basales; lóbulo mediano con ápice redondeado, lóbulo basal subrectangular, más largo que ancho con breve apéndice terminal. (Fig. 2).

DiMORFISMO SEXUAL: ${ }^{\top}$ : Antenas sobrepasan la base pronotal con los últimos 4 artículos; último esternito abdominal visible, con ápice truncado irregularmente a levemente escotado al medio. 9 : Antenas llegan a la base pronotal; último esternito abdominal visible suavemente redondeado.

Material estudiado $\left(60^{\star} 0^{\star}+12 \%\right.$ \% $)$

Cerro Santa Inés, Pichidangui, IV Región. 13/III/2001. Coll.: P. Vidal. Holotipo ( $\sigma^{\top}$ ) depositado en el Museo Nacional de Historia Natural, Santiago, Chile (MNNC); alotipo y 2 paratipos ( ㅇ ㅇ ), misma localidad, fecha y colector, en la colección del Dr. Pedro

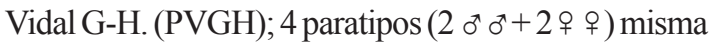
localidad, 2/XII/2000, coll.: P. Vidal: 1 paratipo ( + ) depositado en el Instituto de Entomología, Universidad Metropolitana de Ciencias de la Educación
(UMCE), 1 paratipo ( $\sigma^{\star}$ ) en la colección de T. Moore (TPMR) y 2 paratipos $\left(1 \sigma^{\Uparrow}+1\right.$ \% ) en la colección del Dr. P. Vidal; 1 paratipo ( + ) misma localidad, 10/I/ 2001, coll.: P.Vidal, depositados en la colección del Dr. P. Vidal (PVGH). Alotipo y 1 paratipo colectados el 13/III/2001, fueron colectados bajo corteza, los restantes en hojarasca del mismo bosque.

P. N. Fray Jorge, IV Región, 24/XI/1970, coll.: J. Solervicens (2 우 ㅇ): 2 paratipos depositados en el Museo Nacional de Historia Natural, Santiago, Chile (MNNC), material que ha quedado como referencia del ejemplar rotulado por Germain, actualmente a préstamo en Museo de Praga, Rep. Checa e incorporado como paratipo a la colección del Museo Nacional de Historia Natural (MNNC); 2 paratipos $\left(1 o^{\top}+1\right.$ o $)$ misma localidad, 16/X/1990, coll.: P. Vidal, depositados en su colección (PVGH).

\section{DATOS BIOGEOGRÁFICOS}

Esta especie se encuentra en bosques relictos de la Cordillera de la Costa, del centro-norte del país. En estas áreas, la neblina ("Camanchaca") ha permitido la permanencia de bosques milenarios con flora y fauna muy distintas a sus alrededores. Bosques típicos son Fray Jorge, Talinay y Cerro Santa Inés (Pichidangui). Esta especie vive en detritos vegetales y bajo cortezas (Fig. 5).

Etimología: Nombre dedicado a Marzia Simonetti C., compañera permanente en sus investigaciones y constante colaboradora con Pedro Vidal G-H.

\section{Protoanthicus valenciai $\mathrm{n}$. sp.}

(Figs. 3-4)

Diagnosis: (Holotipo o $\sigma^{\top}$ : Largo $4.8 \mathrm{~mm}$, ancho $1.3 \mathrm{~mm}$. Especie que se destaca por la presencia de dos lóbulos pronotales bien resaltados, producidos por el profundo surco mediano longitudinal (Fig. 3).

CABEZA: Marrón-rojiza, subplana, acortada y ancha; puntuación densa y fina; pubescencia corta castaño; antenas gruesas con segundo artículo subigual que el tercero; frente amplia, no prolongada, ojos pequeños con facetación gruesa. 
Gayana 69(2), 2005
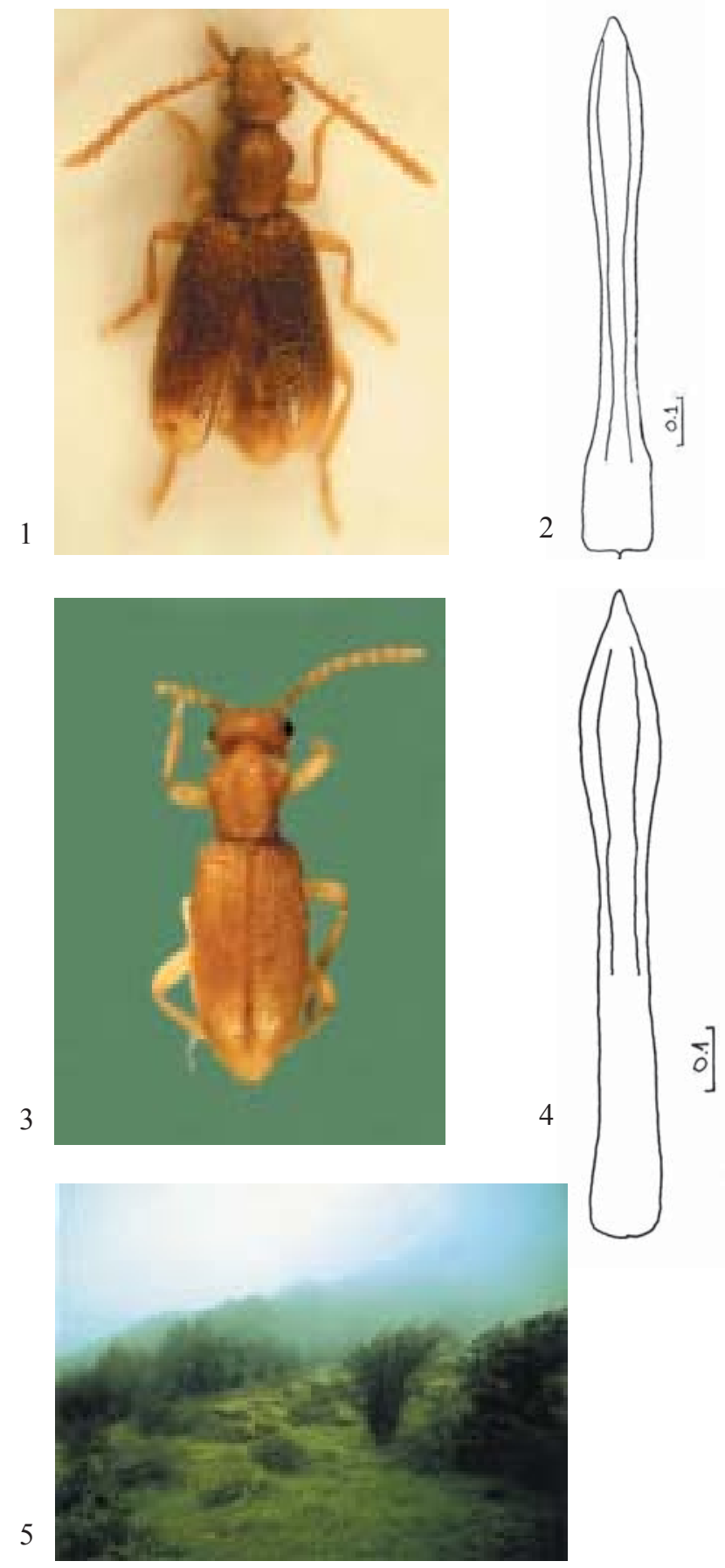

Figuras 1-5. Figs. 1-2: Protoanthicus marziae sp. n., 1, vista dorsal del holotipo ( đ’); 2, edeago, vista dorsal. Figs.3-4: Protoanthicus valenciai sp. n., 3, vista dorsal del holotipo ( $\left.{ }^{\star}\right)$; 4, edeago, vista dorsal. Fig. 5: Hábitat de Protoanthicus marziae sp. n. Cerro Santa Inés, Pichidangui, IV Región.

Figures 1-5. Figs. 1-2: Protoanthicus marziae sp. n., 1, Holotypus, dorsal view ( ๙ $^{\circledR}$ ); 2, Aedeagus, dorsal view. Figs.3-4: Protoanthicus valenciai sp. n., 3, holotypus, dorsal view (o); 4, Aedeagus, dorsal view. Fig. 5: Habitat de Protoanthicus marziae sp. n. Cerro Santa Inés, Pichidangui, IV Región. 
Pronoto: Marrón-rojizo, más largo que ancho; puntuación muy gruesa y densa, lados expandidos hacia la zona basal, dando un aspecto de acinturado; surco mediano fuerte, ancho, profundo cubriendo los $2 / 3$ basales y formando dos lóbulos elevados; pubescencia leonada corta y densa.

Elitros: Marrón-rojizo, mismo color que la cabeza y pronoto, no oscurecidos discalmente, aunque descoloridos en la zona apical; callo humeral inexistente; puntuación redonda y distanciada formando estrías, pubescencia leonada reclinada, larga; lados subparalelos en su mayor parte, apicalmente acortados, dejando a la vista el pigidio, redondeados por separado. Alas vestigiales.

FAZ INFERIOR: Marrón-rojizo brillante, puntuación densa en el esterno; segmentos abdominales oscurecidos en el borde posterior; pilosidad leonada reclinada, epipleuras elitrales ribeteadas, rojizo-oscuro, puntuadas irregularmente, disminuyendo el ancho gradualmente hasta el ápice. Patas testáceo-rojizo, más claras que el resto del insecto.

EDEAGo: Largo y delgado, ensanchado en la mitad del cuarto apical y aguzado hacia el ápice, estrechado después del cuarto apical y gradualmente ensanchado hasta el extremo, incluyendo al lóbulo basal, el cual no posee apéndice terminal; lóbulo mediano con ápice subpuntiagudo.

Dimorfismo SEXUAL: $0^{\star}$ : Ultimo segmento abdominal visible muy levemente redondeado y con borde irregular; antenas largas pasando la base pronotal con los últimos 4 artículos. o : Ultimo segmento abdominal visible, más triangular y suavemente escotado en el ápice: antenas llegan a la base pronotal.

Material estudiado $\left(1 \sigma^{\top}+3\right.$ 우 $)$

El Salto, 320m, Viña, V Región, 8/XI/1992. Coll.: J. Valencia: Holotipo ( $\sigma^{\top}$ ) depositado en la colección del Dr. Pedro Vidal (PVGH).

Jardín Botánico, Viña, V Región. 28/X/1983, coll.: E. Prado (en corteza de Eucaliptus sp.). Alotipo, depositado en el Museo Nacional de Historia Na- tural, Santiago, Chile (MNNC) y un paratipo, misma localidad, fecha y colector, en colección de T. Moore (TPMR):

Etimología: Especie dedicada a nuestro buen amigo y gran estudioso de la entomofauna chilena, Sr. Jorge Valencia J., quien nos dio parte del material para describir la especie.

\section{CONCLUSIONES}

Dado las características de las dos especies que conforman el nuevo género Protoanthicus así como sus genitalias macho que lo asemejan mucho morfológicamente a especies de Nueva Zelandia y Nueva Guinea, que ya nos referimos anteriormente en el análisis de sus genitalias macho, proponemos asignar el género a la subfamilia Lemodinae.

Estas taxa se suman al largo listado de especies animales y vegetales que emergieron del continente Gondwana, como lo evidencian los caracteres anteriormente señalados: genitalias macho simples y muy alargadas, así como el surco mediano del pronoto.

\section{AGRADECIMIENTOS}

Nuestro agradecimiento vaya para quienes nos facilitaron sus materiales para el presente estudio, los Sres. Jorge Valencia, Víctor Manuel Diéguez y Francisco Ramírez, cuyas colecciones se citaron anteriormente y a las instituciones cuyos acrónimos también se señalan en el capítulo Materiales y Método.

\section{BIBLIOGRAFIA}

Abdullah, M. 1969. The natural classification of the family Anthicidae with some ecological and ethological observations. Deutche Entomologische. Zeitschrift, N.F. 16, Helft IV-V:323-366.

Elgueta, M. \& G. Arriagada. 1989. Estado actual del conocimiento de los Coleópteros de Chile (Insecta: Coleoptera). Revista Chilena de Entomología, 17:5-60.

Ross, H. \& J. R. Arnett. 1973. The Beetles of the United States. American Entomological Institute, pp. 747-751. 
Gayana 69(2), 2005

Uhmann, G. 1995. Anthicidae (Insecta: Coleoptera) from New Guinea in the Hornabrook Collection. Journal of the Royal Society of New Zeland, 25(4):517-526.

Werner, F.G. 1958. Some notes on Boheman's Anthicidae from California (Coleoptera). Proceedings of the
Entomological Society of Washington, 60(5): 213-216.

Werner, F. G. 1974. A review of the chilean Anthicidae (Coleoptera). Revista Chilena de Entomología, 8:27-34.

Fecha de recepción: 05/10/04

Fecha de aceptación: 17/08/05 